\title{
Special issue title: Optimization and mathematical modeling in energy systems
}

\author{
Georgios K. D. Saharidis ${ }^{1}$ (D) $\cdot$ Zoi Moza $^{1} \cdot$ Mihalis Golias $^{2}$
}

Received: 21 April 2021 / Accepted: 26 April 2021 / Published online: 16 August 2021

(C) The Author(s), under exclusive licence to Springer-Verlag GmbH Germany, part of Springer Nature 2021

\begin{abstract}
Optimization and mathematical modeling has been successfully used in a variety of applications in the field of Energy Systems. In this Special Volume on optimization and mathematical modeling in energy systems, a variety of research results in different disciplines are included. The electrical energy storage, the resource efficiency of a base camps, the forecasting of energy exports, the wind energy, the optimal management and scheduling of micro grids optimal, the electricity expansion investment problem, the seismic response of a slope earth with embedded pipeline, and the battery deployment strategy, are investigated.
\end{abstract}

Electrical energy storage constitutes a potential candidate capable of regulating the power generation to match the loads via time-shifting. Optimally planning of Electrical energy storage facilities could be an option to meet the increasing requirement of reserves to manage the variability and uncertainty of renewable energy sources. P. Nikolaidis et al. present a methodology for the optimal planning of electrical energy storage facilities involved in contingency-reserve applications. Based on a singlebus model the thermal generation of the power system of Cyprus has been optimally scheduled based on a novel Lagrange Relaxation method for 1 year period without and with electrical energy storage facilities. The derived simulation results showed that improvements exist in profitable return credits when electrical energy storage was integrated with an optimal size of $143.94 \mathrm{MW} / 498.94 \mathrm{MWh}$ based on the worstcase scenario. Finally, the authors performed an uncertainty analysis on net present value, which indicates that $\mathrm{Zn}$-air offers the greatest potential in terms of performance and investment risk.

M. Tous et al. deal with saving measures for laundry, hot water, and potable water production in a military base camp. As the authors explain, improving the resource

Georgios K. D. Saharidis

saharidis@gmail.com

1 Department of Mechanical Engineering, University of Thessaly, Volos, Greece

2 Department of Civil Engineering, University of Memphis, Memphis, TN, USA 
efficiency of base camps is one of the goals for defense authorities not only from cost saving point of view but also from security point of view as suppling units may suffer from enemy assault. The investigated measures were evaluated using the real case of a base with 200 occupants. In the case of laundry measures, it is water re-use and increase of machines' efficiency that are worth applying. Hot water preparation measures consist in recovery of waste heat from exhausts of diesel generators. The measure for potable water production needs specific conditions to be applied, such as the availability of sea or brackish water. However, if the conditions are fulfilled, it is beneficial with a very short payback period less than 2 years, providing at the same time high potential for synergy effects.

N. Alamanis and P. Daloukas investigated the effect of uncertainty related to both the natural heterogeneity of geomaterials and the earthquake shaking characteristics on the seismic performance of slopes. The authors conclude that the effect of the spatial variability of the soil properties on the permanent displacements is very important. The range of variation of the permanent horizontal and vertical displacement is about $\pm 60 \%$ from its mean value. Moreover, N. Alamanis and P. Daloukas found that a slope with spatial variability of soil properties has a $78 \%$ probability to experience larger horizontal displacements than a similar homogeneous slope. The effect of the uncertainty of the frequency content of the earthquake excitation is found to be equally important. For horizontal displacements it may lead to a variation of about $\pm 40 \%$ and for vertical displacements to a variation of $\pm 20 \%$. Finally, the authors develop slope fragility curves in order to be capable to provide the probability of exceedance of various levels of permanent displacement in the horizontal and vertical direction for different levels of peak ground accelerations.

Wind energy is one of the important renewable energy sources that continues to support energy supply to citizens and industrial units. In this frame, improvements, elections and decisions about the choice of wind turbines is still important. Optimization has been used by M. Yörükoğlu and S. Aydın to study the applicability of decision-making method for wind turbine selection problem based on qualitative and quantitative criteria. In the scope of the study, three different modern wind turbines are examined and evaluated based on MULTIMOORA method in terms of six different criteria. The method is based on simple calculation operations which give very effective solutions for decisions like wind turbine selection compare to other methods applied by the authors. The authors show the applicability and validity of their method by considering a real case study and by comparing it with the most widely used method i.e. WASPAS.

Z. Bektas et al. propose a hybrid approach for optimal management of micro grids considering regional conditions and constraints minimizing the amount of power purchased from the main grid. A micro grid is considered in an industrial zone where the power demand per hour has to be matched by scheduling the power loads. An optimization mathematical model is presented and a hybrid solution approach is developed based on genetic algorithms and simulated annealing method. The schedule is programmed using the genetic algorithm and the parameters of the system are optimized by using simulated annealing. The approach has been applied to a four different dates case study with data of Gebze city in Turkey. Mainly, it has appeared that establishing a standalone micro grid is not easy in spite of the 
power surplus during the day. The only option may be storage technologies, which can make it possible.

The effects of two-dimensional variability of soil properties, slope inclination and earthquake excitation characteristics on the development of permanent displacements are investigated from several researchers using random fields created by the Local Average Subdivision method. These random fields of soil properties are characterized by specific mean, variance, cross-correlation coefficients and autocorrelation lengths and used for performing a series of seismic analyses of various slopes by utilizing the FLAC software to calculate finite difference through a Monte Carlo type simulation methodology. The outcomes of an all-encompassing parametric inquiry allow the development of vulnerability curves, based on the permanent horizontal or resultant displacement for several seismic intensity levels. There is also a reference to the mechanical strength and behavior of energy pipelines under stress (bending-buckling) due to the displacement of slopes under seismic forces. Findings clearly show that the influence of spatial variability of soil properties on the ground movement imposed on the embedded pipeline are very important and should be accounted for in the evaluation of its seismic safety. The main conclusions derived from Alamanis et al. show that the two-dimensional variability of soil properties may affect the Factor of Safety of the slopes by approximately $\pm 13 \%$. Additionally, the fact of ignoring the variability of soil properties could lead to permanent earthquake movements that are much smaller than the real ones (up to 53\% smaller in this scenario) resulting in an erroneous (and non-conservative) estimation of seismic behaviour of the slope along with the underground pipeline, located within the mass of the slope. Alamanis et al. show that, due to the slope horizontal and vertical movement, the pipeline in front of the slope suffers a significant compression and an upheaval global buckling. Moreover, it is expected to be subjected to a local buckling at the bottom of the slope; however, this could be specified through a true 3D analysis using finite elements. The results show the curves of vulnerability of slopes against seismic damage and constitute a pretty useful tool for the design of conduits and natural gas pipelines embedded in slopes.

Pappis et al. present an electricity expansion investment outlook (2018-2040) for Paraguay using OSeMOSYS, a cost-optimization modelling framework for medium to long term energy planning, analyzing three electricity demand scenarios under different electricity export prices to Brazil. The study identifies the least-cost power generation mix, future investments and the financial requirements to meet the needs of different demand scenarios. We find that Paraguay will need to invest in hydropower plants, by mainly expanding the capacity of Yacyreta to cover its electricity needs and sustain national electricity exports levels. In the High demand scenario, where the electricity demand could approximately double by 2040, the country's overall electricity exports decrease by $50 \%$ compared to the Reference scenario. Based on the different scenarios examined, the government spends approximately 18.3 - 31.2 billion USD on power plant investments for the period 2018-2040 to cover future electricity demand. The findings could be useful in supporting decision-making concerning socio-economic development pathways in the country.

The seismic response of a slope earth with embedded pipeline is investigated by Alamanis. The results compared with a similar slope with spatial variability of 
soil properties demonstrate that the influence of ground movement imposed on the embedded pipeline is very important and should be accounted for the evaluation of its seismic safety. The study is based on numerical modelling of the soil-pipeline systems, by considering the soil-pipe interaction during seismic shaking. The aim of this study is the investigation of the behaviour of underground energy pipelines under permanent ground displacements. The results demonstrate that the permanent displacements of the heterogeneous slope present large deviations from those of the homogenous slope respectively, when the slopes are subjected to seismic shaking. These movements obviously also affect the behavior of the pipeline within the slope mass. Due to the slope horizontal and vertical movement, the pipeline in front of the slope suffers a significant compression and global buckling. The results presented indicate that transient ground motion of an embedded pipeline in an earth slope may not cause plastic strains on the pipe, if there is no slope failure. This is attributed to the fact that the pipeline flexibility allowed by the two elbows may accommodate small ground movements. By contrast, if the slope experiences failure followed by large displacements (due to strong shaking and small soil strength), the pipe may experience severe global and local buckling. Finally, the magnitude and the spatial distribution of the imposed ground movement may depend significantly on the spatial variability in a zone along the path of the pipeline system.

In the literature, there are limited studies implementing a comparative analysis of "Joint Bidding (JB) strategy" with "battery deployment (BD) strategy". In the existence of balancing responsibility, the comparative analysis of these strategies is the main contribution of M. Özcan et al. The authors analyze the impact of different regulatory regimes, which are set by a regulatory authority, on total income. JBM (Joint Bidding Model), which is the model for joint bidding via different collaboration groups, is developed for the analysis of JB strategy, and BDM (Battery Deployment Model), which is the model covering the deployment of storage technology, is developed for the analysis of BD strategy. The authors analyze the impact of each strategy on total income. According to the analysis, JB strategy, which is sensitive to the regulatory regime, increases the total annual income of the collaboration groups up to $0,65 \%$, and BD strategy seems not feasible and financially viable.

G. Atsalakis et al. apply a novel technique, an integrated neuro-fuzzy controller named PATSOS, for the forecasting of energy exports. The forecasting system is based on two Adaptive Neural Fuzzy Inference Systems (ANFIS) that form an inverse controller. An ANFIS model represents the controller and another ANFIS represents the energy export model that is going to be controlled. ANFIS uses a combination of the least-squares method and the backprobagation gradient descent method to estimate the optimal energy export forecast parameters. Hourly data sets covering a one year period were used to learn and evaluate the proposed system. The forecast accuracy of the proposed technique was evaluated using out of sample tests. The empirical results showed that the system using the bell membership function achieved low values of several type of errors, forecasting well the energy exports.

Finally, A. Nazari et al. using the unit commitment problem develop a formulation for the optimal scheduling of a typical microgrid to minimize costs and emissions. Demand response program (DRP) is utilized to achieve two goals, i.e. to reshape and smooth the load profile, and also for providing reserve capacity along 
with dispatchable units. The optimization is performed by a differential evolution algorithm. The proposed algorithm is called fuzzified modified $\varepsilon$-constraint and deals with multiple criteria decision making in the energy management problem using a non-Pareto based multi-objective solver. The scheduling was performed according to a complete set of operational constraints including spinning and nonspinning reserves. The fuzzified modified $\varepsilon$-constraint method managed to find a solution that optimizes both objectives (cost and emissions) to some extent. This work verifies that the proposed multi-objective approach is able to find an intermediate solution between the two conflicting functions so that as a trade-off each objective is optimized to some extent. The optimal scheduling plan tends to benefit from different electricity prices in day-ahead market and also from the energy storage system to achieve optimal scheduling results. The authors show that in single objective cases (minimization of cost), committing less generating units simultaneously is favorable, as opposed to multiobjective ones in which more available units are tuned on at a lower generating power. Meanwhile, in all cases, the responsive loads are mostly aggregated in off-peak periods to help peak load shaving.

Acknowledgements This research is ongoing with the contribution of the LIFE programme of the European Union-LIFE14 ENV/GR/000611 (GreenYourMove- GYM). GreenYourMove is a European Research Project co-funded by LIFE, the EU financial instrument for the environment. GreenYourMove's main objective is the development and promotion of a co-modal journey application to minimize GHG emission in Europe. Green YourMove develops a multi-modal transport planner (both routing \& ticketing system) considering all kinds of urban public transportation (urban and sub-urban buses, metro, tram, trolley, trains), where the user gets alternative routes combining more than one transport modes if necessary. The routes are the environmentally friendliest ones, since emissions are calculated for different scenarios.

Publisher's Note Springer Nature remains neutral with regard to jurisdictional claims in published maps and institutional affiliations. 